\title{
Economic evaluation of alternative assisted reproduction techniques in management of infertility in Greece
}

This article was published in the following Dove Press journal:

ClinicoEconomics and Outcomes Research

II July 2012

Number of times this article has been viewed

\author{
Vassilis Fragoulakis' \\ Georgia Kourlaba' \\ Basil Tarlatzis ${ }^{2}$ \\ Minas Mastrominas ${ }^{3}$ \\ Nikolaos Maniadakis' \\ 'Department of Health Services \\ Management, National School of \\ Public Health, Athens; ${ }^{2}$ Unit of \\ Human Reproduction, Ist Dept \\ of OB/GYN, Medical School of \\ Aristotle University of Thessaloniki, \\ Thessaloniki; ${ }^{3} \mathrm{Embryogenesis} \mathrm{Centre}$ \\ for Reproductive and Fertility Studies, \\ Athens, Greece
}

Background: The purpose of this study was to compare Gonal- $\mathrm{F}^{\circledR}$, a recombinant follicle-stimulating hormone, with Menopur ${ }^{\circledR}$, a highly purified human menopausal gonadotrophin (hpHMG) in assisted reproduction in Greece.

Methods: A decision tree in combination with a Markov model was used to assess the clinical and economical impact of comparators for up to three consecutive cycles. Transition probabilities were derived from the literature and validated by clinical experts. Cost components were derived from the electronic databases of selected private and public clinics. A probabilistic sensitivity analysis was performed to deal with uncertainty and to construct a cost-effectiveness acceptability curve.

Results: There was a statistically significant difference in favor of the recombinant folliclestimulating hormone arm compared with hpHMG, which was associated with 52 more births (95\% uncertainty interval 26-78, $P=0.001$ ) per 1000 patients. The cost per birth was estimated at $€ 16,906$ and $€ 17,286$ in the recombinant follicle-stimulating hormone and hpHMG arms, respectively. The cost per in vitro fertilization was estimated at $€ 4365$ in the recombinant folliclestimulating hormone arm and $€ 3815$ in the hpHMG arm, indicating a difference of $€ 550$. The incremental cost per birth for recombinant follicle-stimulating hormone versus hpHMG was estimated at $€ 14,540$, while the incremental cost per life-year was estimated at $€ 175.41$.

Conclusion: Recombinant follicle-stimulating hormone may represent a cost-effective choice compared with hpHMG when used for ovarian stimulation for a pharmacoeconomic point of view in the Greek public health care setting. However, it must be noted that in clinical practice both agents may be used together to increase the number of follicles, oocytes, embryos, and/or pregnancies in treated patients, an approach which has not been evaluated in Greece or reported in the literature due to obvious limitations.

Keywords: cost-effectiveness, Gonal- $\mathrm{F}^{\circledR}$, Menopur ${ }^{\circledR}$, highly purified human menopausal gonatotrophin

\section{Introduction}

Infertility is a condition which, while not a direct threat to physical health, is associated with significant psychological distress and impaired social well-being. Notably, its prevalence is increasing for various social and demographic reasons. ${ }^{1,2}$ The most common risk factors for infertility include age, smoking, alcohol use, excess weight, caffeine intake, occupational and environmental risk exposure, emotional factors, and chronic illnesses, such as diabetes or thyroid disease. ${ }^{3}$ The prevalence ranges significantly in different countries and there is a paucity of reliable data. It is estimated that about $10 \%-15 \%$ of couples experience infertility at some point in their reproductive years and between $42 \%-76 \%$ of them seek treatment. ${ }^{1-6}$
Correspondence: Nikolaos Maniadakis Department of Health Services and Management, National School of Public Health, 196 Alexandras Ave,

Athens II52I, Greece

Tel +30 2I 06467097

Fax +30 21 3201 0194

Email nmaniadakis@esdy.edu.gr 
In this context, the demand for and use of assisted reproductive technologies has steadily increased in Europe, especially in the last decade. ${ }^{7}$ Since the birth of the first in vitro fertilization baby more than 30 years ago, an estimated 3.5 million children have been born worldwide after treatment with assisted reproductive technology. ${ }^{8}$ In conventional in vitro fertilization treatment, gonadotrophins are administered in order to stimulate the ovarian cycle. Follicle-stimulating hormone is universally recognized as the key driver of ovarian follicle growth and maturation. It is also well established that folliclestimulating hormone and luteinizing hormone play separate but complementary roles in folliculogenesis. ${ }^{9}$ The "two cell, two gonadotropin theory" suggests that interaction between follicle-stimulating hormone and luteinizing hormone is crucial for appropriate folliculogenesis and oocyte maturation. Throughout most of the follicle's development, luteinizing hormone responsiveness is restricted to thecal cells that are differentiated in the follicular preantral stage. During folliculogenesis, androgens are produced in the thecal cells of antral follicles in response to luteinizing hormone stimulation. ${ }^{9}$

Highly purified human menopausal gonatotrophin (hpHMG) has been the traditional therapy, but in recent years, recombinant gonadotropin (follicle-stimulating hormone) preparations have become available, and novel stimulation protocols have been developed for their use. Gonal- $\mathrm{F}^{\circledR}$ (follitropin alfa for injection) is a human follicle-stimulating hormone preparation of recombinant DNA origin, which consists of two noncovalently linked, nonidentical glycoproteins designated as the $\alpha$ - and $\beta$-subunits. Its safety and efficacy in relation to older urinary gonadotrophin preparations is well documented in the literature. ${ }^{10-15}$

The cost-effectiveness of the two treatments under evaluation has been investigated in other studies reported in the literature but with inconsistent results. ${ }^{16-18}$ Specifically, recombinant follicle-stimulating hormone was estimated as a cost-effective treatment strategy in ovulation induction prior to in vitro fertilization with an incremental cost per additional pregnancy of 2583 pounds in one study. ${ }^{17}$ However, another study showed that recombinant folliclestimulating hormone was a more costly option, with similar efficacy for one or three treatment cycles. ${ }^{18}$ Moreover, a study conducted in the US estimated the cost-effectiveness of the agents under several hypothetical drug price scenarios. A Markov model and Monte Carlo simulation techniques were used to model the possible outcomes during three treatment cycles. The authors concluded that, despite its greater cost per unit dose, recombinant follicle-stimulating hormone is cost-effective in relation to hpHMG, because of its greater clinical efficacy relative to its comparator. ${ }^{19}$ Another economic study showed a 9.4\% reduction in the overall therapy cost per baby born in favor of recombinant follicle-stimulating hormone against hpHMG. ${ }^{11}$ However, contrasting results were obtained in a study which evaluated the cost-effectiveness of the two treatments for intrauterine insemination cycles in patients with polycystic ovary syndrome. This study showed that the urinary preparation was more cost-effective, due mainly to the difference of the drug cost per IU. ${ }^{20}$ The above evidence makes it clear that the cost-effectiveness of these agents may be different in different settings, so it is important to consider the studies in the context within which they were undertaken and remember that it may be challenging to extrapolate.

As in many other countries, infertility is a multidimensional problem in Greece, with social, economic, and cultural implications, and has reached significant proportions in recent years, with an increasing number of couples seeking treatment. Health care resources are scarce, especially in the context of the present economic and financial crisis, and must be allocated in ways which maximize the return of the investment for individuals and society. The number of couples using this approach to achieve a pregnancy per annum is large and the cost of therapy is high. Hence, an economic evaluation was undertaken in order to assess recombinant follicle-stimulating hormone in comparison with hpHMG in terms of cost-effectiveness in the Greek setting. The present paper presents the results of this analysis.

\section{Materials and methods Perspective of economic evaluation}

The aim of the present study was to conduct an economic evaluation comparing Gonal-F (Merck Serono) which is a newer recombinant therapy, with an older urinary alternative, Menopur ${ }^{\circledR}$, (Ferring Lægemidler), used for ovarian stimulation in Greece. The majority of infertility treatments in Greece are carried out in the private sector, in the in vitro fertilization departments of private hospitals or specialized clinics across the country. The cost of therapy is mainly covered by social security sickness funds, but patients may have to contribute to cover some elements of therapy in many cases. Thus, this analysis was carried from the perspective of social security sickness funds and patients together. In this context, only direct health care costs carried by patients and social security sickness funds were considered. These include the cost of drugs, hospital and physician visits, and examinations. Other forms of economic burden associated with infertility treatment, such as absence from 
work, travelling expenses, or even psychological distress, were not considered in the present analysis.

\section{Type of analysis and costing methodology}

The management of patients and the delivery of therapy is somewhat complex and there are many different pathways. Thus, a modeling approach was utilized. Specifically, a decision tree in combination with a Markov model was adapted and customized from the literature to assess the clinical and economical outcomes of the two comparators for the case of Greece..$^{21}$ A representation of the Markov model developed is depicted in Figure 1. It was developed with feedback from medical experts to reflect the local management of patients in the context of the Greek health care system. In short, the structure of the model is identical for both the recombinant and the urinary branch and the differences relate only to the cost of the resources expended and the transition and outcome probabilities in different nodes of the model. The model simulates the progression of patients from the moment they start therapy, to various states based on specified probabilities collected from the literature. The first cycle is a complete treatment cycle with the aim of obtaining egg cells, fertilizing them, and performing embryo transfer. This process may end up in an actual pregnancy and birth, or may fail, in which case there could be a completely new treatment cycle, a frozen embryo transfer in the event that it was possible to cryopreserve embryos, or individuals may decide to stop the in vitro fertilization effort. Transition probabilities for the various states were obtained from the modeling study used as the basis to develop the present one and were originally based on a large observational study undertaken in Germany with 24,764 assisted reproductive cycles in total. ${ }^{21,22}$ The probabilities were verified by a panel of local experts and are presented in Table 1. Furthermore, the probabilities presented in this table were assigned in the model beta distributions with $10 \%$ coefficients of variance, in order to deal with uncertainty.

Given that the payment policy by social security sickness funds covers three cycles per patient and taking into account the in vitro fertilization protocols applied in Greece, the time horizon of the model was set up for three consecutive in vitro fertilization cycles. In terms of treatment costs, the May 2011 price bulletin issued by the Ministry of Health was used to retrieve the prices of in vitro fertilization drugs. The cost was $€ 0.361$ per IU $/ \mathrm{mL}$ in the case of recombinant follicle-stimulating hormone and much lower at $€ 0.118$ in the case of hpHMG. Based on the aforementioned observational study and expert advice, it was assumed that the average dose per in vitro fertilization cycle was 2325 IU in the first case and 2828 in the second case..$^{21,22}$ In other words, higher doses were assigned for hpHMG. Furthermore, based on resource inputs derived from the electronic databases of three selected clinics in the country and expert advice, the cost of initial examinations and preparations was estimated at $€ 600$,

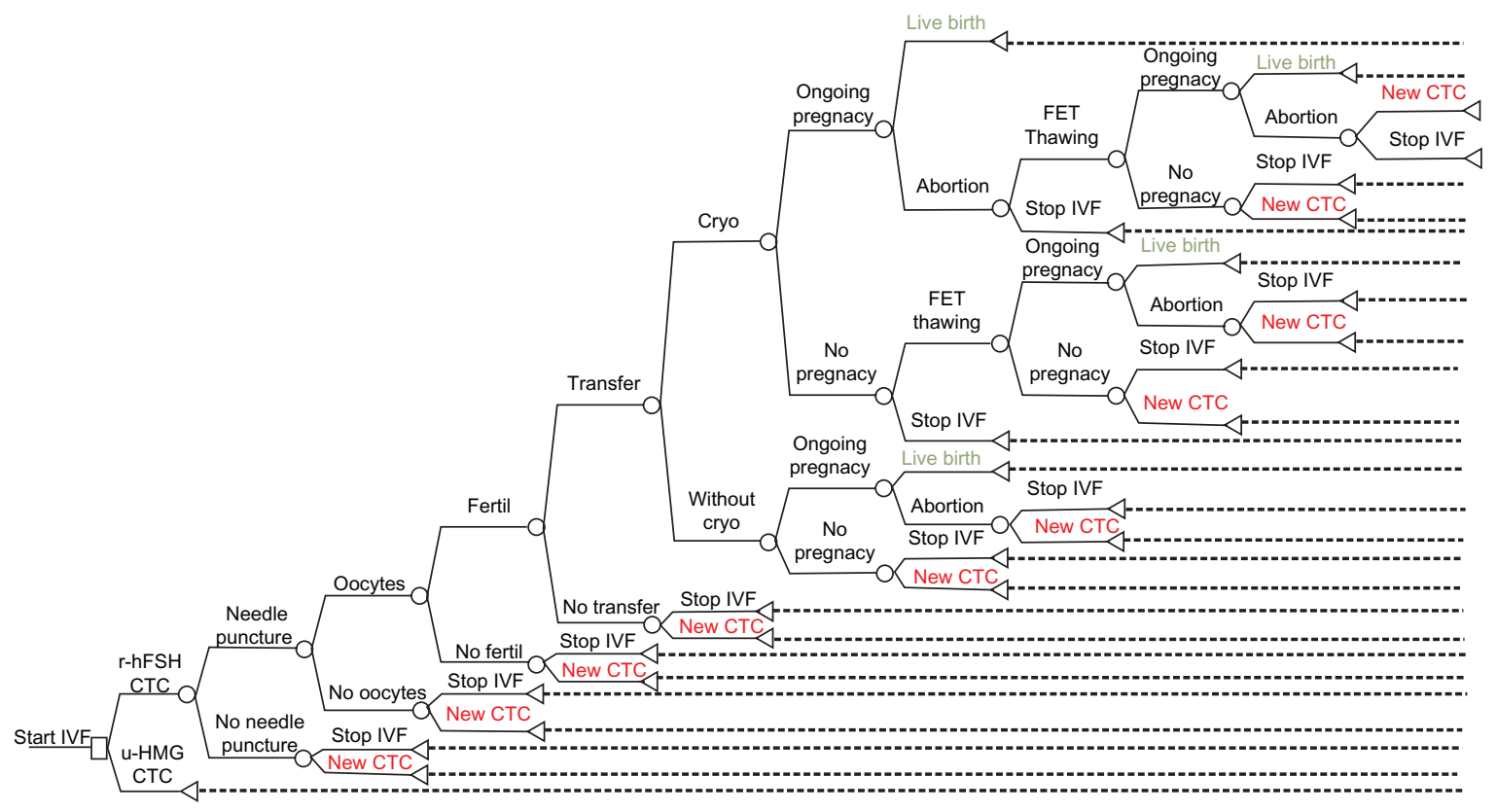

Figure I Structure of model.

Abbreviations: CTC, complete treatment cycle; IVF, in vitro fertilization; r-hFSH, recombinant human follicle-stimulating hormone; U-HMG, urinary human menopausal gonadotrophin; FET, frozen embryo transfer. 
Table I Probabilities of occurrence in the model*

\begin{tabular}{lll}
\hline & $\begin{array}{l}\text { rFSH } \\
\text { (Gonal-F }^{\circledR} \text { ) }\end{array}$ & $\begin{array}{l}\text { hpHMG } \\
\text { (Menopur }^{\circledR} \text { ) }\end{array}$ \\
\hline Needle puncture after treatment & $94.70 \%$ & $93.00 \%$ \\
Needle puncture to deliver oocytes & $99.10 \%$ & $98.90 \%$ \\
No needle puncture to new CTC & $68.38 \%$ & $68.85 \%$ \\
Oocytes to fertilization & $94.99 \%$ & $94.52 \%$ \\
No oocytes to new CTC & $68.38 \%$ & $68.85 \%$ \\
Fertilization to transfer & $98.39 \%$ & $99.05 \%$ \\
No fertilization to a new CTC & $68.38 \%$ & $68.85 \%$ \\
Transfer to cryopreservation & $80.00 \%$ & $80.00 \%$ \\
No transfer to a new CTC & $68.38 \%$ & $68.85 \%$ \\
Cryopreservation to pregnancy & $30.00 \%$ & $27.79 \%$ \\
Without cryopreservation to pregnancy & $30.00 \%$ & $27.79 \%$ \\
Ongoing pregnancy live birth & $76.28 \%$ & $74.36 \%$ \\
No pregnancy to FET thawing & $95.00 \%$ & $95.00 \%$ \\
No pregnancy to a new CTC & $68.38 \%$ & $68.85 \%$ \\
Abortion to a new CTC & $68.38 \%$ & $68.85 \%$ \\
Abortion to FET thawing & $95.00 \%$ & $95.00 \%$ \\
FET thawing to pregnancy & $17.20 \%$ & $14.10 \%$ \\
Pregnancy to live birth after FET thawing & $65.10 \%$ & $58.60 \%$ \\
\hline
\end{tabular}

Note: *Beta distribution was assigned to all probabilities with a $10 \%$ coefficient of variation.

Abbreviations: CTC, complete treatment cycle; FET, frozen embryo transfer; $\mathrm{rFSH}$, recombinant follicle-stimulating hormone; hpHMG, highly purified human menopausal gonatotrophin.

the cost of oocytes at $€ 300$, the cost of needle puncture at $€ 400$, the cost of fertilization at $€ 950$, the cost of transfer at $€ 750$, the cost of cryopreservation at $€ 300$, and the cost of frozen embryo transfer thawing at $€ 1000$. All the above figures were assigned log-normal distributions to deal with uncertainty in probabilistic analysis.

Ovarian hyperstimulation syndrome is an uncommon but potentially life-threatening complication in the fertilization process, characterized by ovarian enlargement, high serum sex steroids, and extravascular fluid accumulation, primarily in the peritoneal cavity. In severe cases, hypotension, increased coagulability, reduced renal perfusion, and in some cases fatalities, have been reported. ${ }^{23}$ The present economic model accounts for the case of ovarian hyperstimulation syndrome. Specifically, the ovarian hyperstimulation syndrome base rate applied in the model is set at $5 \%$ for both comparators, based on the literature and expert opinion. ${ }^{10,15,21-27}$ The cost of managing a case of ovarian hyperstimulation syndrome, based on data from various private clinics across the country, was estimated on average at $€ 700$. A log-normal distribution was assumed for probabilistic analysis, based on sampling variability of cost and a beta distribution for the ovarian hyperstimulation syndrome rate, assuming 5\% variability. The cost per pregnancy and per birth is estimated as the ratio of the mean (bootstrapped) cost per patient over the mean (bootstrapped) success rate, ie, mean expected pregnancies and births.

\section{Uncertainty}

The nature of the present analysis and the issue in hand requires undertaking of extensive uncertainty analyses. The management of patients is complex with many contingencies, particularly in reality and consequently in the model. The probabilities associated with the various outcomes and pathways are subject to variation and the same applies to patient treatment costs. The data used also come from different sources and are subject to uncertainty, which is why computer-simulated clinical and economic models are used in pharmacoeconomic evaluations. In this context, to evaluate the robustness of the results obtained and to deal with uncertainty, all input parameters were associated with probability distributions and Monte Carlo simulation was then undertaken for probabilistic analysis. In every simulation, the value for each parameter is determined independently and at random from the prespecified distributions, and the results are recalculated. The Monte Carlo simulation was run in total 5000 times and the results of the probabilistic analysis were used to calculate cost-effectiveness acceptability curves. Sensitivity analysis was not undertaken because most of the data are based on observational analyses, and most of the assumptions in the model are identical for the two therapies. Hence, the results and the conclusions will not change significantly if one starts varying them proportionally.

\section{Results}

Results are shown in detail in Table 2 and Figures 2 and 3. The main medical outcomes of the model are pregnancies and births per therapy arm within the three treatment cycle horizons studied. The results presented are based on averages and statistics derived from the 5000 simulations. According to the analysis, there is a statistically significant difference in favor of recombinant follicle-stimulating hormone compared with hpHMG in terms of births, ie, the main outcome. In particular, it is predicted that there will be 52 more births (95\% uncertainty interval [UI] 26-78, $P=0.001$ ) per 1000 patients treated. Put in other words, the probability of birth with recombinant follicle-stimulating hormone is $44.6 \%$ (95\% UI 42.5-46.6) whilst with hpHMG it is 39.4\% (95\% UI $37.5-41.2$ ), ie, a difference of 5.2\% (95\% UI 2.6-7.8). The recombinant therapy is more expensive due to the cost of drugs. Total treatment cost per patient in the recombinant follicle-stimulating hormone arm is $€ 7540.67$ (95\% UI $7284.82-7787.18)$ and in the hpHMG arm is $€ 6812.10(95 \%$ UI 6554.70-7060.81), a difference of $€ 728.58$ (95\% UI 434.53-1018.80) in favor of the older treatment. 
Table 2 Cost and medical outcomes per therapy arm per patient treated based on 5000 simulations

\begin{tabular}{|c|c|c|c|c|c|c|c|c|c|c|}
\hline \multirow[t]{2}{*}{ Cost $(€)$} & \multicolumn{3}{|c|}{ Gonal-F $^{\circledR}(\mathrm{rFSH})$} & \multicolumn{3}{|c|}{ Menopur $^{\circledR},($ hpHMG) } & \multicolumn{3}{|c|}{ Difference } & \multirow[t]{2}{*}{$P$ value $^{\mathrm{a}}$} \\
\hline & Mean & 95\% LUI & 95\% UUI & Mean & 95\% LUI & 95\% UUI & Mean & 95\% LUI & 95\% UUI & \\
\hline Initial cost & $1036.5 \mid$ & 983.75 & 109.44 & 1071.35 & 1015.38 & 1128.60 & -34.84 & -65.53 & -3.74 & 0.027 \\
\hline Cost of oocytes & 486.87 & 457.94 & 514.85 & 492.95 & 462.93 & 523.00 & -6.08 & -32.59 & 20.24 & 0.652 \\
\hline Cost of needle puncture & 655.22 & 616.14 & 694.07 & 664.80 & 626.59 & 705.22 & -9.58 & -44.41 & 24.21 & $0.58 \mathrm{I}$ \\
\hline Cost of fertilization & 1463.62 & 1365.45 & 1564.39 & 1474.44 & 1373.48 & 1576.36 & -10.82 & -97.95 & 73.03 & 0.805 \\
\hline Cost of transfer & 1137.46 & 1062.62 & 1214.77 & 1153.95 & 1077.64 & 1230.96 & -16.50 & -86.73 & 52.30 & 0.640 \\
\hline Cost of cryopreservation & 363.81 & 339.76 & 387.16 & 369.08 & 344.90 & 392.75 & -5.27 & -27.53 & 16.72 & 0.639 \\
\hline Cost of FET thawing & 888.23 & 820.17 & 958.84 & 927.06 & 858.14 & 999.80 & -38.84 & -95.75 & 17.38 & 0.178 \\
\hline Total cost of procedure & 6031.71 & 5789.79 & 6270.71 & 6153.64 & 5900.42 & 6396.97 & -121.93 & $-4|I .4|$ & 154.26 & 0.398 \\
\hline Cost of drugs & 1448.48 & 1395.32 & 1503.66 & 595.94 & 572.72 & 619.21 & 852.54 & 794.81 & 909.59 & 0.000 \\
\hline Cost of adverse events & 60.48 & 58.21 & 62.84 & 62.52 & 59.29 & 65.93 & -2.04 & -4.99 & 0.86 & 0.174 \\
\hline Total effectiveness & 7540.67 & 7284.82 & 7797.18 & 6812.10 & 6554.69 & 7060.81 & 728.58 & 434.53 & 1018.792 & 0.001 \\
\hline Pregnancies & 0.593 & 0.565 & 0.619 & 0.546 & 0.520 & 0.570 & 0.047 & 0.011 & 0.081 & 0.009 \\
\hline Births & 0.446 & 0.425 & 0.466 & 0.394 & 0.375 & 0.412 & 0.052 & 0.026 & 0.078 & 0.001 \\
\hline
\end{tabular}

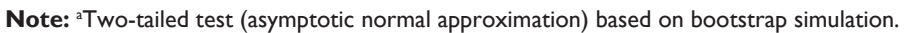

Abbreviations: LUI, lower uncertainty interval; UUl, upper uncertainty interval; FET, frozen embryo transfer; rFSH, recombinant follicle-stimulating hormone; hpHMG, highly purified human menopausal gonatotrophin.

Hence, the cost per pregnancy is estimated at $€ 12,715.13$ (95\% UI 12,345.48-13,131.31) in the case of recombinant follicle-stimulating hormone and $€ 12,478.15$ (95\% UI $12,134.23-€ 12,832.35)$ in the case of hpHMG. The mean difference was estimated at $€ 238.32$ (95\% UI 142.22-671.32, $P=0.254$ ), which failed to demonstrate a statistical significant difference between the two treatments. The cost per birth was $€ 16,906.22$ (95\% UI 16,347.12-17,516.35) and $€ 17,286.84$ (95\% UI 16,740.12-17,845.33) in the recombinant folliclestimulating hormone arm and hpHMG arm, respectively. The difference is $-€ 379.85$ (95\% UI 1024.45-315.78, $P=0.269$ ) which again is not statistically significant. The cost per in vitro fertilization was $€ 4365.12$ (95\% UI 4205.69-4506.32) in the recombinant follicle-stimulating hormone and $€ 3815.69$ (95\% UI 3661.41-3953.55) in the hpHMG arm, indicating a difference at $€ 550.55$ (95\% UI 365.32-730.22, $P=0.001$ ). Importantly, the incremental cost per birth for recombinant follicle-stimulating hormone relative to hpHMG was estimated at $€ 14,540.45$ (95\% UI 10,509.78-21,868.96). The mean life expectancy at birth in Greece is 79.92 years and, in this light, the cost per life-year gained with recombinant follicle-stimulating hormone is estimated at $€ 175.41$

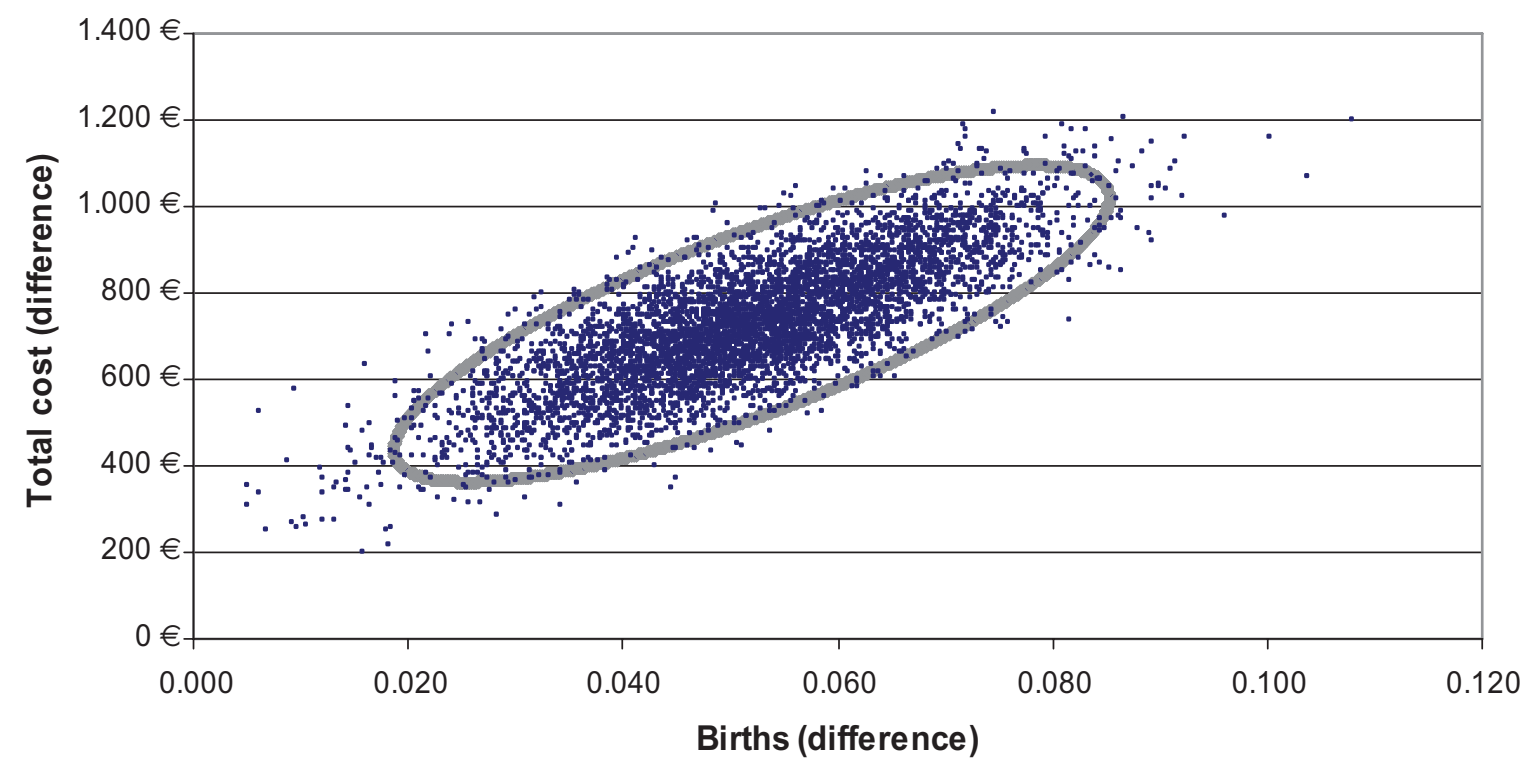

Figure 2 Scatter plot based on probabilistic analysis.* Note: *Ellipse represents the $95 \%$ uncertainty intervals. 


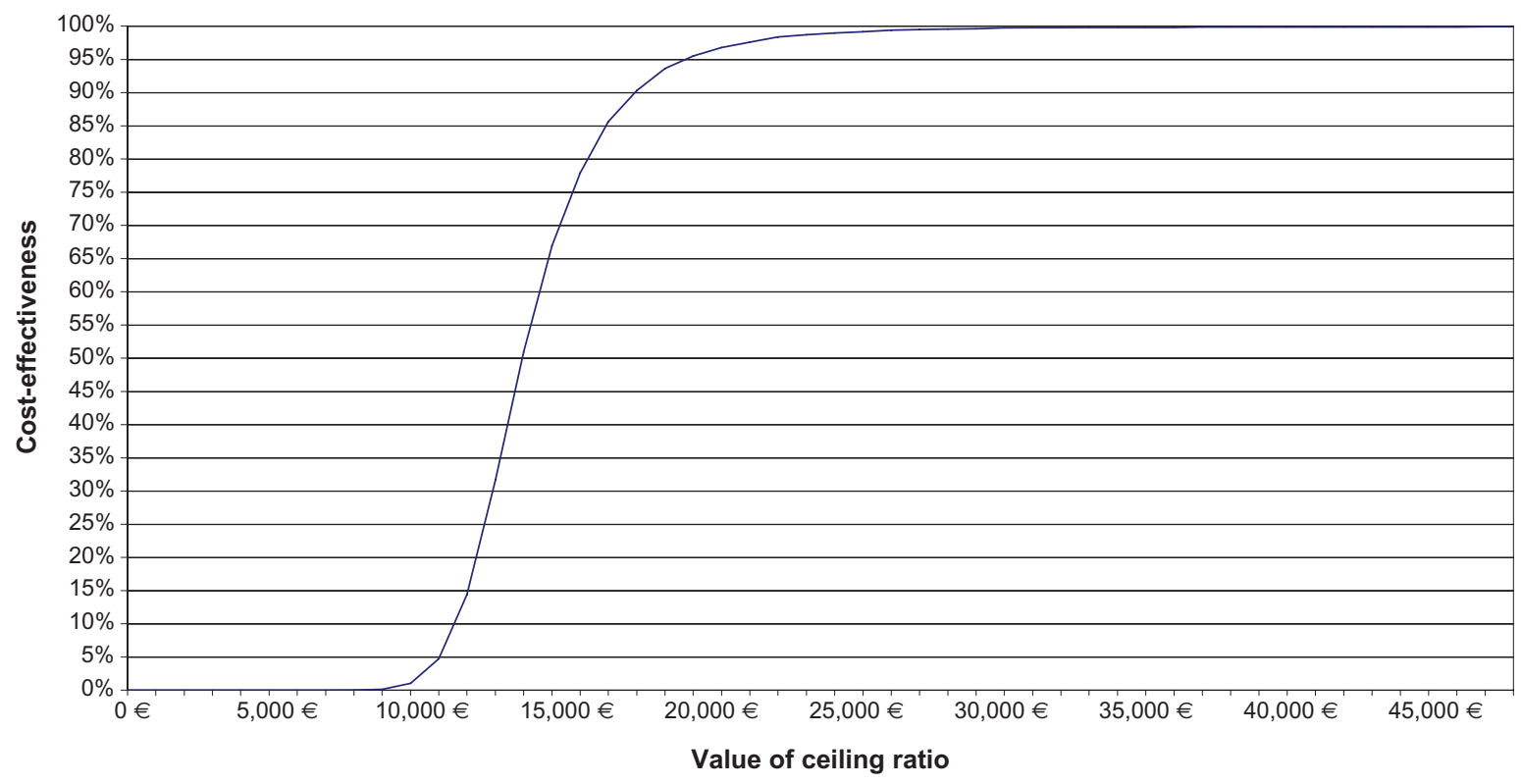

Figure 3 Cost-effectiveness acceptability curve of recombinant follicle-stimulating hormone versus highly purified human menopausal gonatotrophin.

(95\% UI 163.44-213.17). This figure is well below the figure of $€ 40,000$ to $€ 50,000$ per life-year gained, used as a threshold in many jurisdictions, and also below the figure of three times the local per capita gross domestic product, which is often cited by the World Health Organization. ${ }^{28}$

Furthermore, the results of the simulation were used to plot the distribution of differences in costs and effects in the cost-effectiveness plane. Figure 2 shows the 5000 pairs of differences in treatment cost and effect (birth) from the simulation, where all the stochastic parameters received values from the log-normal and beta distributions. It is seen that all the differences fell into the upper right quadrant, indicating that recombinant follicle-stimulating hormone was more expensive but also more effective than hpHMG in all cases. Whether the points in the scatter plot indicate that recombinant follicle-stimulating hormone is cost-effective in relation to its comparator depends on the willingness to pay per birth achieved. A convenient way of illustrating this is by the cost-effectiveness acceptability curve (Figure 3), which depicts the probability of a treatment being costeffective at different willingness-to-pay thresholds. In this particular case, the probability that the incremental cost per birth with recombinant follicle-stimulating hormone reaches $95 \%$ at $€ 20,000$.

\section{Discussion}

Understanding the relative benefits and costs of alternative strategies for infertility management is important in order to ensure that patients receive effective but also economically efficient care. In the present study, a comparison of recombinant follicle-stimulating hormone and hpHMG was undertaken using a decision tree with a local adaptation of an established Markov probabilistic model. The selected alternatives are widely used in Greece. The comparison assumed an average of up to three treatment cycles, an assumption based on the aforementioned model and local economic and clinical data. The analysis indicated that recombinant follicle-stimulating hormone is associated with greater cost and more effectiveness, and probabilistic analysis showed that recombinant follicle-stimulating hormone is cost-effective with a high level of statistical significance.

Several studies have examined the two therapies considered here, and the results are somewhat inconsistent due to differences in patient management, costs of therapy, and the methodology used in the evaluations. ${ }^{1,2,19,29-32}$ The sort of analysis undertaken here requires use of economic modeling. In this context, it is important that the structure of the model reflects reality and that clinical and economic data are reliable. The model used was taken from the literature and was adapted with clinical experts to reflect the management of patients in the present local setting. In terms of effectiveness, a meta-analysis of three randomized controlled trials undertaken back in 2003 concluded that no significant differences existed between the two treatment groups in ovulation rate, pregnancy rate, miscarriage, and multiple pregnancy rates. ${ }^{10,15,25,26}$ Also, a more recent meta-analysis indicated that there was insufficient evidence to support superiority of 
hpHMG over recombinant follicle-stimulating hormone in in vitro fertilization. ${ }^{27}$ Clinical trials may have high internal but low external validity and they are concerned with efficacy as opposed to effectiveness. On the other hand, well designed, large, multicenter, pragmatic observational studies may have high internal as well as external validity, and are concerned with effectiveness, which is more interesting from an economic policy perspective. In this context, a large representative observational study from Germany was used to quantify the effectiveness of the two therapies based on expert advice which was considered appropriate for the Greek setting in terms of patient management and outcomes. ${ }^{22}$ This study was preferred due to a large number of treatment cycles and the representative nature of the data used, and the economic results are similar to those attained for Germany.

Overall, the present analysis suffers from drawbacks and limitations common to all studies using similar methodology. It does not represent experimental research, but instead is based on a model populated from data reported in the literature and on various assumptions, and thus may suffer from biases. To limit possible sources of bias, standard recommendations were followed. Thus, a systematic review and assessment of the evidence was performed in collaboration with clinician experts, and stochastic analysis was used to draw robust conclusions. However, this methodology cannot substitute for direct real-life comparisons between these treatments. Another limitation of the study is that the use of hpHMG as a possible useful adjunct to recombinant follicle-stimulating hormone is not considered in the present model. Nonetheless, it must be noted that in clinical practice both agents may be used together to increase the number of follicles, oocytes, embryos, and/or pregnancies in treated patients. In addition, it was assumed for simplicity that there was no difference in dosage between patient groups, such as "normal infertile couples" or women with "polycystic ovarian syndrome". Hence, only an average dose for each agent was used in the model, according to expert advice.

The results have to be considered strictly in the Greek setting and on the basis of present time resources and drug prices. If any of the underlying parameters change, so may the results and conclusions of the analysis. Finally, we confined the analysis to sickness funds and patients, and not to society overall. There may also be other societal, economic, and clinical outcomes which may have to be investigated in the future.

\section{Conclusion}

The results of the present study indicate that recombinant follicle-stimulating hormone may represent a cost-effective choice compared with hpHMG for ovarian stimulation from a pharmacoeconomic point of view in the setting of the Greek national health care system. However, it must be noted that in clinical practice both agents may be used together to increase the number of follicles, oocytes, embryos, and/or pregnancies in treated patients, an approach that has not been evaluated locally or in the relevant literature, for obvious reasons.

\section{Disclosure}

The authors report no conflicts of interest.

\section{References}

1. Daya S, Ledger W, Auray JP, et al. Cost-effectiveness modelling of recombinant FSH versus urinary FSH in assisted reproduction techniques in the UK. Hum Reprod. 2001;16(12):2563-2569.

2. Wechowski J, Connolly M, McEwan P, Kennedy R. An economic evaluation of highly purified HMG and recombinant FSH based on a large randomized trial. Reprod Biomed Online. 2007;15(5):500-506.

3. Roupa Z, Polikandrioti M, Sotiropoulou P, et al. Causes of infertility in women at reproductive age. HSJ. 2009;3(2):80-87 Available at: http:// www.hsj.gr/volume3/issue2/324.pdf.

4. Gnoth C, Godehardt E, Frank-Herrmann P, Friol K, Tigges J, Freundl G. Definition and prevalence of subfertility and infertility. Hum Reprod. 2005;20(5):1144-1147.

5. Benagiano G, Bastianelli C, Farris M. Infertility: a global perspective. Minerva Ginecol. 2006;58(6):445-457.

6. Boivin J, Bunting L, Collins JA, Nygren KG. International estimates of infertility prevalence and treatment-seeking: potential need and demand for infertility medical care. Hum Reprod. 2007;22(6):1506-1512.

7. Andersen AN, Goossens V, Ferraretti AP, et al. Assisted reproductive technology in Europe, 2004: results generated from European registers by ESHRE. Hum Reprod. 2008;23(4):756-771.

8. Wennerholm UB, Söderström-Anttila V, Bergh C, et al. Children born after cryopreservation of embryos or oocytes: a systematic review of outcome data. Hum Reprod. 2009;24(9):2158-2172.

9. Durnerin CI, Erb K, Fleming R, et al. Effects of recombinant LH treatment on folliculogenesis and responsiveness to FSH stimulation. Hum Reprod. 2008;23(2):421-426.

10. Loumaye E, Martineau I, Piazzi A, et al. Clinical assessment of human gonadotrophins produced by recombinant DNA technology. Hum Reprod. 1996;11 Suppl 1:95-107.

11. Revelli A, Poso F, Gennarelli G, Moffa F, Grassi G, Massobrio M. Recombinant versus highly-purified, urinary follicle-stimulating hormone (r-FSH vs HP-uFSH) in ovulation induction: a prospective, randomized study with cost-minimization analysis. Reprod Biol Endocrinol. 2006;4:38

12. Balen A, Platteau P, Andersen AN, Devroey P, Helmgaard L, Arce JC. Highly purified FSH is as efficacious as recombinant FSH for ovulation induction in women with WHO Group II anovulatory infertility: a randomized controlled non-inferiority trial. Hum Reprod. 2007;22(7):1816-1823.

13. Platteau P, Andersen AN, Balen A, et al. Similar ovulation rates, but different follicular development with highly purified menotrophin compared with recombinant FSH in WHO Group II anovulatory infertility: a randomized controlled study. Hum Reprod. 2006;21(7):1798-1804.

14. Szilagyi A, Bartfai G, Manfai A, Koloszar S, Pal A, Szabo I. Low-dose ovulation induction with urinary gonadotropins or recombinant follicle stimulating hormone in patients with polycystic ovary syndrome. Gynecol Endocrinol. 2004;18(1):17-22.

15. Yarali H, Bukulmez O, Gurgan T. Urinary follicle-stimulating hormone (FSH) versus recombinant FSH in clomiphene citrate-resistant, normogonadotropic,chronic anovulation: a prospective randomized study. Fertil Steril. 1999;72(2):276-281. 
16. Ola B, Afnan M, Sharif K, Hammadieh N, Papaioannou S, Coomarasamy A. Cost-effectiveness of recombinant versus urinary follicle stimulating hormone; whose point of view? Acta Obstet Gynecol Scand. 2001;80(12):1141-1142.

17. Sykes D, Out HJ, Palmer SJ, van Loon J. The cost-effectiveness of IVF in the UK: a comparison of three gonadotrophin treatments. Hum Reprod. 2001;16(12):2557-2562.

18. Hatoum HT, Keye WR Jr, Marrs RP, Walton SM, Marshall DC. A Markov model of the cost-effectiveness of human-derived folliclestimulating hormone (FSH) versus recombinant FSH using comparative clinical trial data. Fertil Steril. 2005;83(3):804-807.

19. Silverberg K, Schertz J, Falk B, Beresniak A. Impact of urinary FSH price: a cost-effectiveness analysis of recombinant and urinary FSH in assisted reproduction techniques in the USA. Reprod Biomed Online. 2002;5(3):265-269.

20. Gerli S, Casini ML, Unfer V, Costabile L, Mignosa M, Di Renzo GC. Ovulation induction with urinary FSH or recombinant FSH in polycystic ovary syndrome patients: a prospective randomized analysis of costeffectiveness. Reprod Biomed Online. 2004;9(5):494-499.

21. Schoffsci O, Sowietzki S, Ludwig M. Decision tree analysis to determine the cost-effectiveness of r-FSH compared with u-HMG in treatment cycles with assisted reproduction techniques. Pharmacoeconomics. 2007;5(1):29-39.

22. Ludwig M, Rabe T, Bühler K, Diedrich K, Felberbaum R. Efficacy of recombinant human FSH in comparison to urinary hMG following a long down-regulation protocol - an analysis of 24,764 ART cycles in Germany. Journal für Reproduktionsmedizin und Endokrinologie. 4/2004;1(2):82-90. German.

23. Delvigne A, Kostyla K, De Leener A, et al. Metabolic characteristics of women who developed ovarian hyperstimulation syndrome. Hum Reprod. 2002;17(8):1994-1996.

24. Andersen AN, Devroey P, Arce JC. Clinical outcome following stimulation with highly purified $\mathrm{hMG}$ or recombinant $\mathrm{FSH}$ in patients undergoing IVF: a randomized assessor-blind controlled trial. Hum Reprod. 2006;21(12):3217-3227.
25. van Wely M, van der Veen F. Meta-analysis of recombinant FSH and urinary-derived gonadotrophins for IVF or ICSI. Hum Reprod. 2003;18(7):1554-1555.

26. Coomarasamy A, Afnan M, Cheema D, van der Veen F, Bossuyt $\mathrm{PM}$, van Wely $\mathrm{M}$. Urinary hMG versus recombinant FSH for controlled ovarian hyperstimulation following an agonist long down-regulation protocol in IVF or ICSI treatment: a systematic review and meta-analysis. Hum Reprod. 2008;23(2):310-315.

27. Jee BC, Suh CS, Kim YB, Kim SH, Moon SY. Clinical efficacy of highly purified hMG versus recombinant FSH in IVF/ICSI cycles: a meta-analysis. Gynecol Obstet Invest. 2010;70(2):132-137.

28. Zhao FL, Yue M, Yang H, Wang T, Wu JH, Li SC. Willingness to pay per quality-adjusted life year: is one threshold enough for decisionmaking? Results from a study in patients with chronic prostatitis. Med Care. 2011;49(3):267-272.

29. Wechowski J, Connolly M, Schneider D, McEwan P, Kennedy R. Cost-saving treatment strategies in in vitro fertilization: a combined economic evaluation of two large randomized clinical trials comparing highly purified human menopausal gonadotropin and recombinant follicle-stimulating hormone alpha. Fertil Steril. 2009;91(4): 1067-1076.

30. Romeu A, Balasch J, Ruiz Balda JA, et al. Cost-effectiveness of recombinant versus urinary follicle-stimulating hormone in assisted reproduction techniques in the Spanish public health care system. J Assist Reprod Genet. 2003;20(8):294-300.

31. Silverberg K, Daya S, Auray JP, et al. Analysis of the cost effectiveness of recombinant versus urinary follicle-stimulating hormone in in vitro fertilization/intracytoplasmic sperm injection programs in the United States. Fertil Steril. 2002;77(1):107-113.

32. Connolly M, De Vrieze K, Ombelet W, Schneider D, Currie C. A cost per live birth comparison of HMG and rFSH randomized trials. Reprod Biomed Online. 2008;17(6):756-763.
ClinicoEconomics and Outcomes Research

\section{Publish your work in this journal}

ClinicoEconomics \& Outcomes Research is an international, peerreviewed open-access journal focusing on Health Technology Assessment, Pharmacoeconomics and Outcomes Research in the areas of diagnosis, medical devices, and clinical, surgical and pharmacological intervention. The economic impact of health policy and health systems

\section{Dovepress}

organization also constitute important areas of coverage. The manuscript management system is completely online and includes a very quick and fair peer-review system, which is all easy to use. Visit http://www.dovepress.com/testimonials.php to read real quotes from published authors. 